\title{
Análisis de datos espaciales del Índice de Necesidades Básicas Insatisfechas Caso de estudio: Región Andina
}

\author{
Spatial Data Analysis of the Unsatisfied Basic Needs Index. \\ Study Case: Andean Region
}

Carolina Aponte Gómez ${ }^{1}$

Elkin Mauricio Romero Aroca ${ }^{2}$

Luis Fernando Santa Guzmán ${ }^{3}$

Para citar este artículo, utilice el nombre completo así:

\section{Resumen}

El trabajo consistió en estimar un Modelo de Regresión Espacial de Tipo Beta para determinar el Índice de Necesidades Básicas Insatisfechas en la región Andina de Colombia, considerando variables basadas en el censo poblacional de 2005. Los datos empleados fueron obtenidos del Sistema de Información Geográfica para la Planeación y Ordenamiento Territorial. El efecto de dependencia espacial fue incorporado con un modelo mixto regresivo autorregresivo espacial. Los resultados obtenidos mostraron que el modelo presenta un buen ajuste, resultando significativas algunas de las variables valoradas, y, además, destacaron la necesidad de contemplar la presencia de autocorrelación espacial en la estimación de los parámetros. Por otra parte, cabe destacar la posibilidad de contar con una aproximación al valor del índice a partir de las variables consideradas sin tener que realizar un censo poblacional. El trabajo fue desarrollado empleando los softwares R 3.2.1 y ArcGis 10.2.2.

1 Estudiante Ingeniería Catastral y Geodesia, Universidad Distrital Francisco José de Caldas, caponteg18@gmail.com

2 Estudiante Ingeniería Catastral y Geodesia, Universidad Distrital Francisco José de Caldas, mauricio.romero83@gmail.com

3 MSc. en Geomática, Universidad Nacional de Colombia. Profesor Asistente Universidad Distrital Francisco José de Caldas, Ifsantag@unal.edu.co 
Palabras clave: Índice de Moran, Modelo de regresión tipo beta, NBI (Necesidades básicas Insatisfechas), Pobreza, Región andina, Rezago espacial.

\section{Abstract}

The present paper uses a Beta Type Spatial Regression Model, to determine the Unsatisfied Basic Needs' Index in the Andean Region of Colombia, considering the explanatory variables based on the population census of 2005. The data used were obtained from Geographic Information System for Planning and Land Management. The effect of spatial dependence was incorporated, through the setting of a regressive autoregressive space mixed model. The results showed that the model has a good fit; some of the variables valued ending up being significant, which pointed out the need to contemplate the presence of the spatial autocorrelation in the models parameters' estimation. On the other hand, the possibility of having an approximation to the value of the index, from the variables considered, without having to carry out a population census should be noted. The work was developed using the software R 3.2.1 and ArcGIS 10.2.2.

Keywords: Moran index, Beta Type Regression Model, NBI (Unsatisfied Basic Needs), Poverty, Andean Region, Lag Spatial. 


\section{Introducción}

Los estudios sobre el índice de Necesidades Básicas Insatisfechas (NBI) en Colombia se han llevado a cabo a partir de la información de los censos generales que desarrolla el Departamento Administrativo Nacional de Estadística (DANE), el último de los cuales es del año 2005. El índice de NBI ha sido obtenido con un modelo de variables exógenas, denominadas indicadores simples, que determinan si las necesidades básicas de los habitantes de un municipio son cubiertas (DANE, 2006); pero dichos indicadores no son empleados para un análisis de la espacialidad del fenómeno, y no se precisa realmente el porqué se dan dichas condiciones de pobreza y de calidad de vida.

Debido a que la pobreza es un fenómeno muy complejo, su estudio debe ser afrontado desde diferentes ángulos (Fresneda, 2007), entendiendo que "el ingreso es necesario, pero no es suficiente para conseguir el bienestar" (González, 2011). En la actualidad se emplean diferentes tipos de índices para reflejar o identificar la población realmente pobre, los cuales se apoyan en la información que recolecta la variable en estudio, por lo que no se busca reemplazarla, sino que se trata de hacerla más robusta y constante (Baltazar, Grillo, \& Karpf, 2007).
Al cambiar por variables obtenidas constantemente en el tiempo, mediante la colaboración de datos públicos de las entidades gubernamentales del país, se puede obtener una base de datos para analizar e implementar un Modelo Espacial de Regresión Beta con los factores que expliquen las condiciones de pobreza en tiempo real (Ferrari \& CribariNeto, 2004), y así determinar los lugares donde falta la intervención de las administraciones estatales, de tal forma que sea equitativa la asignación de recursos de acuerdo con el nivel de pobreza presente en cada municipio.

Es importante plantear un modelo de datos espaciales que permita explicar las NBI a partir de un conjunto de factores asociados a cada municipio, ya que, en este momento, los resultados que se obtienen de este índice no cuentan con un análisis actual o físico de la situación en tiempo real (Casas y Bohórquez, 2005). El modelo debe permitir recolectar la información de las variables independientes, sin necesidad de implementar un censo poblacional, en vista de que este índice es la base para la distribución de los recursos destinados a los municipios anualmente en cada plan de desarrollo (Baltazar y Carpe, 2007); índice que se debe generar y actualizar cada 4 años de acuerdo con lo estipulado en la Ley 152 de 1994. 


\section{Materiales y Métodos}

\section{1 Área de estudio}

El trabajo se desarrolló en la Región Andina, una de las cinco regiones naturales de Colombia, conformada por los departamentos de Antioquia, Boyacá, Caldas, Cauca, Cesar, Cundinamarca, Huila, Nariño, Norte de Santander, Putumayo, Quindío, Risaralda, Santander, Tolima y Valle del Cauca (Andrade et al., 2003), mostrados en la Figura 1. Alberga esta región el $67.17 \%$ de la población de Colombia y, además, corresponde a casi 16 millones de habitantes según el censo de 2005 (DANE, 2006). Esta zona abarca un territorio de 282.450 km2 (24,8\% del total del país). Bogotá concentra más del $16 \%$ de la población y cada departamento supera el millón de habitantes, indicando la gran densidad poblacional en esta región, que cuenta con gran diversidad y desigualdad en aspectos económicos y sociales (Salazar, 2010).

\subsection{Materiales}

Se construyó la base de datos con los datos obtenidos en el Sistema de Información Geográfica para la Planeación y el Ordenamiento Territorial [SIGOT], que reúne la información de 16 autoridades e instancias en el Sistema de Planeación del Territorio, a nivel nacional, regional y local (IGAC, 2008). La base de datos se muestra en la Tabla 1, donde se relacionan las variables utilizadas, con la sigla correspondiente utilizada para el desarrollo del modelo, una breve descripción de cada una, su rango de valores y la fuente o autoridad de la información en el SIGOT.

La base de datos fue organizada empleando el software ArcGis 10.2.2 y exportada en formato Shapefile [.shp], para realizar el análisis espacial, y en formato Texto [.txt], para la manipulación de los datos en el software de procesamiento. Con esta base de datos de 1.124 datos se filtraron los departamentos pertenecientes a la Región Andina y se eliminaron los municipios con datos omitidos, dando como resultado 760 observaciones para realizar el modelo. El software de procesamiento utilizado fue $\mathrm{R}$ versión 3.2.1 de código abierto, que permite la libertad de uso (González \& Daffara, 2000), para llevar a cabo la estimación del Modelo Espacial de Regresión Beta con paquetes como: Car, Rcmdr, aplpack, RODBC, scatterplot3d, betareg, vegan, spacemakeR, maptools, spdep, ape, ade4, deldir y GeoXp (Cribari-Neto \& Zeileis, 2010).

\subsection{Métodos}

El análisis de la información obtenida en la base de datos se llevó a cabo por medio de la calificación de las variables dependiente e independientes; de 


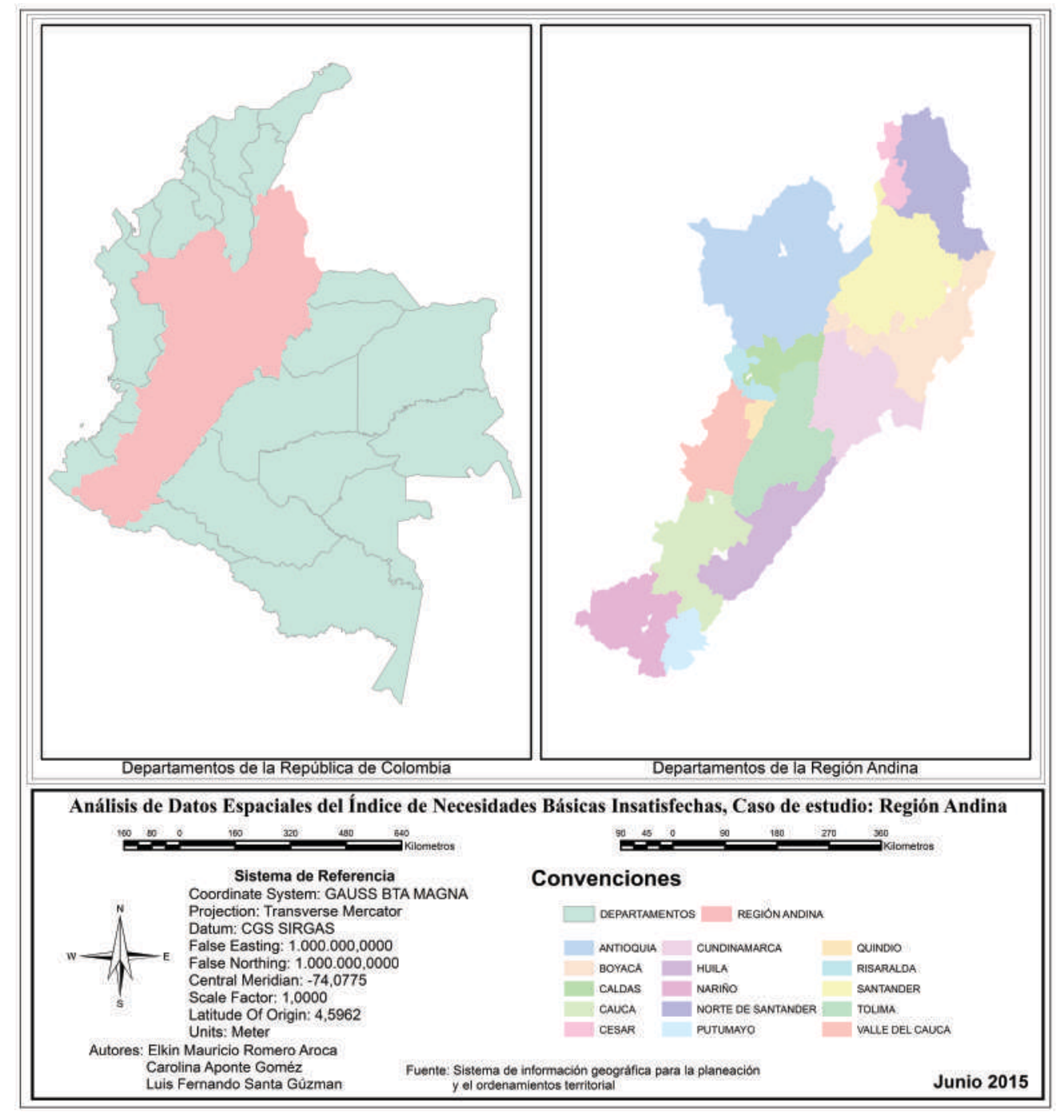

Figura 1. Delimitación Región Andina

Fuente: Elaboración propia

esta forma fue posible establecer los tipos de datos de cada variable, su naturaleza matemática, la escala de medida y sus niveles (Rangos de valores). Después se realizó la descripción de datos, partiendo de las posiciones de las observaciones como puntos en el espacio, es decir, definiendo los diferentes vectores para realizar el análisis de datos multivariantes (Peña, 2002); luego, se importaron los datos al software $\mathrm{R}$ para realizar el análisis exploratorio, identificando las variables cuantitativas y cualitativas 
Tabla 1. Descripción de variables

\begin{tabular}{|c|c|c|c|c|}
\hline Variable & Sigla & Descripción & $\begin{array}{c}\text { Niveles / Rango de } \\
\text { valores }\end{array}$ & Fuente \\
\hline $\begin{array}{l}\text { Necesidades } \\
\quad \text { Básicas } \\
\text { Insatisfechas }\end{array}$ & NBI & $\begin{array}{l}\text { Indicador que deter- } \\
\text { mina el porcentaje } \\
\text { de carencias de una } \\
\text { población }\end{array}$ & {$[0-1]$} & $\begin{array}{l}\text { Departamento } \\
\text { Administrativo } \\
\text { Nacional de } \\
\text { Estadística }\end{array}$ \\
\hline $\begin{array}{l}\text { Logaritmo de } \\
\text { densidad de } \\
\text { población }\end{array}$ & LOGDP & $\begin{array}{l}\text { Número de habitantes } \\
\text { sobre el área muni- } \\
\text { cipal por kilómetro } \\
\text { cuadrado }\end{array}$ & {$[-0,05-4,14]$} & $\begin{array}{l}\text { Departamento } \\
\text { Administrativo } \\
\text { Nacional de } \\
\text { Estadística }\end{array}$ \\
\hline $\begin{array}{l}\text { Magnitud de } \\
\text { la inversión } \\
\text { municipal }\end{array}$ & MIM & $\begin{array}{l}\text { Porcentaje del total } \\
\text { de recursos anuales } \\
\text { destinados al muni- } \\
\text { cipio por kilómetro } \\
\text { cuadrado }\end{array}$ & {$[0-1]$} & $\begin{array}{l}\text { Departamento } \\
\text { Nacional de } \\
\text { Planeación }\end{array}$ \\
\hline $\begin{array}{l}\text { Generación de } \\
\text { recursos pro- } \\
\text { pios (esfuerzo } \\
\text { fiscal) }\end{array}$ & $\begin{array}{l}\text { GRPI, GPRB, } \\
\text { GRPM, GRPS }\end{array}$ & $\begin{array}{l}\text { Recursos destinados } \\
\text { a financiar el activo } \\
\text { fijo presentado por el } \\
\text { municipio }\end{array}$ & $\begin{array}{c}\text { GRPI: }<10 \\
\text { Insuficiente, GRPB: } \\
\text { 10,1 - } 20 \text { Bajo, } \\
\text { GRPM: } 20,1-40 \\
\text { Medio GRPS: }>40,1 \\
\text { Suficiente }\end{array}$ & $\begin{array}{l}\text { Departamento } \\
\text { Nacional de } \\
\text { Planeación }\end{array}$ \\
\hline $\begin{array}{l}\text { Logaritmo } \\
\text { del consumo } \\
\text { promedio de } \\
\text { energía por } \\
\text { habitante }\end{array}$ & LOGCPENR & $\begin{array}{l}\text { Consumo promedio } \\
\text { por habitante sector } \\
\text { residencial }\end{array}$ & {$[2,36-5,74]$} & $\begin{array}{l}\text { Superintendencia } \\
\text { de Servicios } \\
\text { Públicos } \\
\text { Domiciliarios }\end{array}$ \\
\hline $\begin{array}{l}\text { Tasa de afilia- } \\
\text { ción régimen } \\
\text { contributivo }\end{array}$ & TCONTVO & $\begin{array}{l}\text { Porcentaje de per- } \\
\text { sonas afiliadas al } \\
\text { sistema de salud vin- } \\
\text { culadas laboralmente }\end{array}$ & {$[0-1]$} & $\begin{array}{c}\text { Ministerio de } \\
\text { Protección Social }\end{array}$ \\
\hline $\begin{array}{l}\text { Logaritmo } \\
\text { del ingreso } \\
\text { público per } \\
\text { cápita }\end{array}$ & LOGIPP & $\begin{array}{l}\text { Total de recursos } \\
\text { anuales en la entidad } \\
\text { territorial sobre su } \\
\text { población total }\end{array}$ & {$[2,082-3,435]$} & $\begin{array}{l}\text { Departamento } \\
\text { Nacional de } \\
\text { Planeación }\end{array}$ \\
\hline $\begin{array}{l}\text { Cobertura en } \\
\text { educación }\end{array}$ & COBEDU & $\begin{array}{l}\text { Número de estudian- } \\
\text { tes por nivel sobre } \\
\text { la población según el } \\
\text { rango de edad corres- } \\
\text { pondiente }\end{array}$ & {$[28,28-210,85]$} & $\begin{array}{l}\text { Ministerio de } \\
\text { Educación }\end{array}$ \\
\hline $\begin{array}{c}\text { Déficit de } \\
\text { vivienda total }\end{array}$ & DEFCVT & $\begin{array}{l}\text { Número de hogares } \\
\text { que presentan caren- } \\
\text { cias habitacionales so- } \\
\text { bre el total de hogares } \\
\text { del municipio X100 }\end{array}$ & {$[0-1]$} & $\begin{array}{l}\text { Departamento } \\
\text { Administrativo } \\
\text { Nacional de } \\
\text { Estadística }\end{array}$ \\
\hline
\end{tabular}

Fuente: Elaboración propia 
(Canavos, 1988), (Wooldridge, 2010). Cómo el fenómeno de las NBI presenta datos espaciales con variación discreta (Schabenberger \& Gotway, 2004), es decir, datos lattice de regiones irregulares, se usó el análisis exploratorio mediante métodos de estadística descriptiva (Noel, 1993); también se elaboró un análisis exploratorio espacial multivariado para buscar autocorrelación, y la relación entre las variables endógena y exógenas (Montgomery \& Runger, 2002).

Para la estimación del Modelo Espacial de Regresión Beta se realizó primero un modelo sin efectos espaciales, donde se utilizó el paquete BETAREG del software $\mathrm{R}$ (Cribari-Neto \& Zeileis, 2010), para estimar los parámetros y examinar las distintas funciones de enlace, utilizando los criterios de información bayesiana [BIC], parámetro de precisión y pseudo-R2 (Gujarati \& Porter, 2010). Teniendo la función de enlace seleccionada, se realizaron las pruebas estadísticas basadas en el contraste de razón de verosimilitudes, los índices de Moran y de Geary y los factores de inflación de la varianza [VIF], para verificar los supuestos del modelo de multicolinealidad y autocorrelación, y su bondad de ajuste. Además, se analizaron los residuales para hallar los datos influyentes y atípicos, utilizando los puntos de Laverage generalizados, la distancia de COOK y los valores
Hat (Canavos, 1988); (Peña, 2002); (Fernandez, 2008); (Dobson, 2002).

Al encontrar autocorrelación espacial, fue necesario construir el rezago espacial de cada una de las variables para ser incluidas en la estimación del modelo; esto se hizo utilizando la selección de matrices de vecindad mediante las coordenadas principales de matrices de contigüidad [PCNM] (Valcarce \& Serrano, 2000); para así realizar de nuevo las pruebas de bondad de ajuste y evaluación de los supuestos. Para la identificación de la estructura de la dependencia espacial se estableció la mejor matriz de ponderación espacial; para ello se tuvieron en cuenta criterios de contigüidad física, de gráficas y basados en distancias (Lance \& Carol, 2004), y así se realizó el cálculo de los índices globales y locales de autocorrelación espacial, tales como el de Moran y el de Geary (O)Sullivan \& Unwin, 1943). Finalmente, se analizaron los residuales del modelo final y se compararon los valores observados y estimados de la variable respuesta. El modelo espacial con dependencia sustantiva resultante proporcionó una buena aproximación al sistema real de las NBI.

\section{Marco referencial}

Para identificar los hogares pobres se puede aplicar el denominado método directo, que evalúa directamente si 
los hogares han logrado satisfacer sus necesidades básicas, encuestándolos sobre los bienes y servicios de que disponen; otra alternativa es el método indirecto, que mide los ingresos y los gastos. En los dos casos se define si estas características son suficientes para tener un buen nivel de vida. En el primer método se relaciona el bienestar con el consumo efectivamente realizado, mientras que el segundo relaciona la posibilidad de realizar consumo. El primer método es conocido como el de Necesidades Básicas Insatisfechas (NBI), introducido por la Comisión Económica para América Latina y el Caribe (CEPAL) en los años ochenta, con la finalidad de analizar la información adquirida en censos demográficos y de vivienda (Feres \& Mancero, 2001).

Se describen dos pasos principales en la medición de la pobreza, los cuales se deben abordar: identificar la población en condición de pobreza entre la población total, y construir una medida numérica para dicha condición (Sen, 1976). Se relacionan los métodos indirectos para adquirir tal información y el detalle de adquisición para tomarlo como marco en las actuales medidas de pobreza. Mediante medidas multidimensionales se tiene una alternativa que identifica la población en condición de pobreza y la pobreza agregada, con un enfoque práctico; además, en cada medida donde se desarrollan índices se descompone si se exhibe el nivel de pobreza agregada. Se destaca la importancia de las técnicas multidimensionales, especialmente en la etapa de identificación por 'dual cut off', o doble corte, que define a una persona pobre de acuerdo con sus privaciones (Alkire \& Foster, 2011).

En Latinoamérica, la metodología de las NBI ha sido importante para identificar en la población ciertas carencias críticas y la caracterización de la pobreza. Se define la pobreza como «la situación de aquellos hogares que no logran reunir, en forma relativamente estable, los recursos necesarios para satisfacer las necesidades básicas de sus miembros» (Feres \& Mancero, 2001). Como medida, la pobreza ha sido usada para propósitos de diagnóstico social, y ha servido de apoyo para identificar la población en donde se desarrollan programas sociales en distintos países. Se considera que el índice de NBI debe ser actualizado casi cada 20 años, debido a los cambios en los campos de bienes y servicios, considerados cruciales para la población; obteniendo un concepto subjetivo y un lapso extenso para su estimación. Simultáneamente, se logra un mejoramiento de la metodología, para tener un conjunto de medidas que muestren la intensidad de la pobreza y la desigualdad en el interior de los pobres, pero también se obtiene una 
adecuación al marco de derechos humanos y a las políticas de descentralización; además, de una inclusión de nuevas temáticas en el censo ejecutado (Fresneda, 2007).

En Colombia, el Gobierno nacional ha tenido avances concretos en estimar la cantidad de población en condición de pobreza, por lo que se encuentran indicadores adicionales como el Índice de Condiciones de Vida [ICV] y el índice del gasto social, SISBEN, los cuales miden el bienestar o calidad de vida. Estos indicadores también pueden ser adaptados para medir la pobreza en la población, aunque cualquier indicador de pobreza debe satisfacer las propiedades axiomáticas propuestas por Sen (1976).

También se plantea que en cada individuo hay presentes factores institucionales, económicos, sociales y políticos, entre otros, que afectan de forma independiente las tasas de pobreza del hogar. Se propone un modelo general para un solo período, el cual relaciona la tasa de pobreza de las familias como variable dependiente, explicada por un vector con varias variables de características económicas y estructurales, un vector de variables con características demográficas y un vector de variables con características sociales y políticas (Rupasingha \& Goetz, 2007). Se realiza la selección de las variables independientes con toda la información disponible para la población y "se realiza la selección del conjunto de variables independientes espacializadas que caracterizan los municipios en las diferentes áreas para su posterior análisis en el índice de NBI" (Benson, Chamberlin, \& Rhinehart, 2005).

\section{Resultados y Discusión}

\subsection{Variable endógena}

El índice de NBI en la Región Andina presenta la mayor concentración de municipios en el intervalo 20\%-40\%, indicando que esta región presenta un bajo nivel de este indicador; además, el comportamiento de los datos muestra una distribución asintótica a la derecha. En el diagrama de distribución empírica, mostrado en la Figura 2, se puede observar que la variable sigue una distribución beta con parámetros de forma $\alpha=2,953$ y $\beta=4,875$. Al hacer la prueba de Kolmogorov-Smirnov se encontró que no hay evidencia estadística suficiente para rechazar la hipótesis nula de que sigue una distribución beta . También se muestra evidencia de la presencia de algunos datos atípicos indicados en el diagrama de caja en la Figura 2.

El coeficiente de variación da un valor de $43.49 \%$, indicando que los datos tienen una dispersión media 

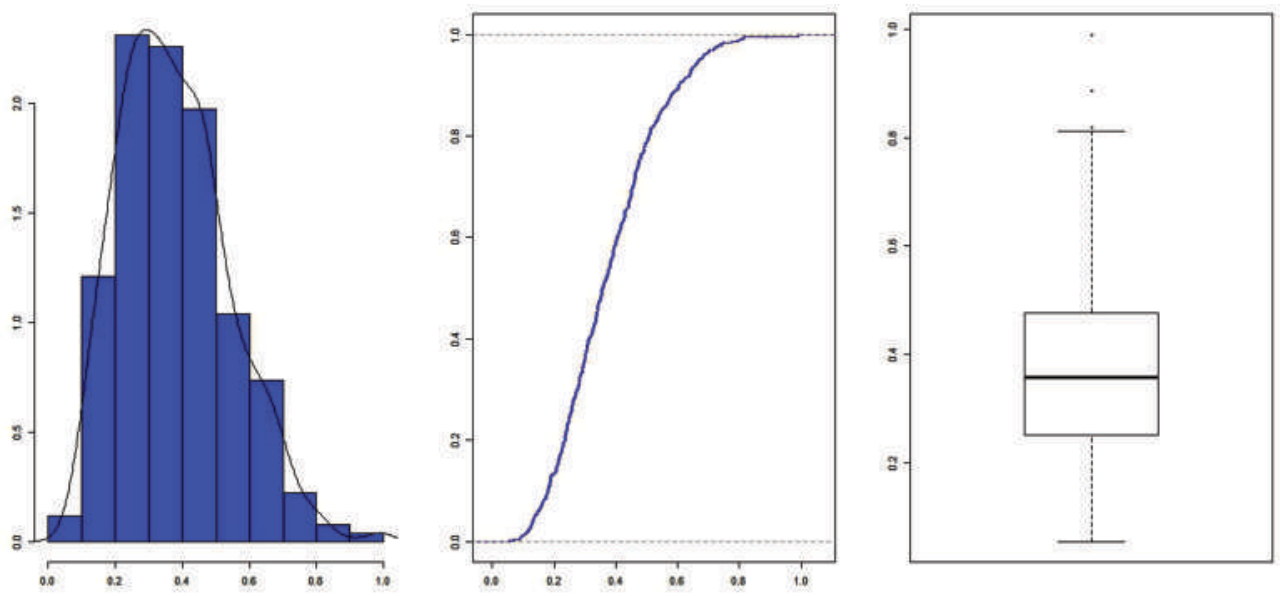

Figura 2. Histograma, diagrama de distribución empírica y diagrama de caja de la variable explicada.

Fuente: Elaboración propia

respecto a su promedio. El análisis espacial permite identificar la interacción del NBI en cada municipio; usando la prueba de Mantel como medida de dependencia espacial se comprobó estadísticamente que las observaciones de esta variable en 2005 están correlacionadas espacialmente, donde el estadístico de prueba, con un nivel de significancia del 5\%, rechaza la hipótesis nula.

Mediante las pruebas de Moran y Geary se comprueba que existe una autocorrelación espacial global de los datos, ya que la hipótesis nula es rechazada en ambos casos ( ; ); en la Figura 3 se muestra el gráfico de autocorrelación de Moran respecto a la matriz de pesos espaciales del criterio de contigüidad de vecinos relativos, dando como resultado una dependen- cia espacial positiva que indica que los municipios de escasos recursos están rodeados de otros municipios en la misma condición, mientras que los municipios con mayor capacidad de recursos están contiguos de otros municipios en igual situación.

Con la prueba de Moran para autocorrelación espacial mostrada en al mapa de índice local de autocorrelación espacial [LISA], de la Figura 3 , se encontraron agregaciones de pobreza en las zonas norte y sur de la Región Andina, siendo los municipios Almaguer-Cauca, Chita-Boyacá, San Calixto-Norte de Santander y La Vega-Cauca con mayor NBI. Las agregaciones de bajas NBI se ubican al oriente y occidente de la región, donde se encuentran los departamentos de Cundinamarca, Quindío y 

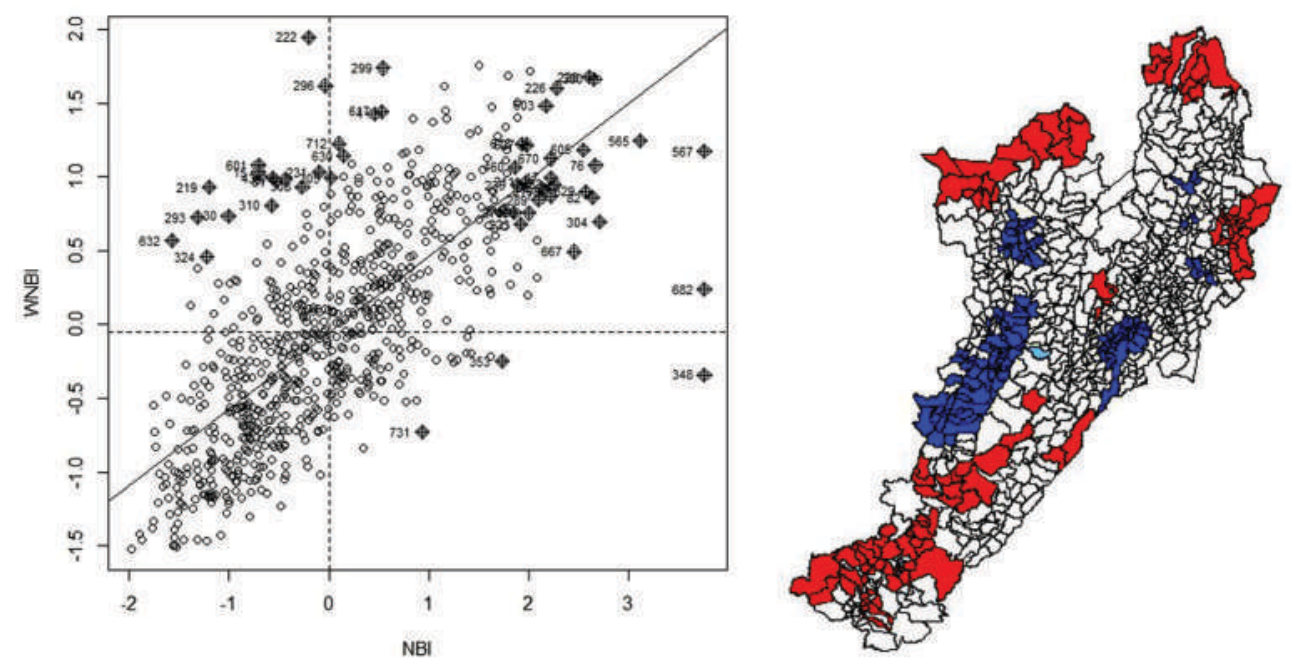

Figura 3. Dispersograma de Moran y Mapa LISA Fuente: Elaboración propia

Valle del Cauca, como se muestra en el Figura 4.

Se tiene que para los criterios de vecindad evaluados, el criterio de ocho vecinos más cercanos presentó el menor criterio de información Akaike [AIC], por lo que fue el seleccionado para hallar el rezago espacial de la variable endógena.

\subsection{Variables exógenas}

En las variables independientes IPP, DP y CPENR se realiza una transformación usando el logaritmo natural para linealizarlas y así expresar su relación con la variable endógena, como se muestra en los diagramas de dispersión en la Figura 5; también se encontró una relación directamente proporcional con DEFCVT y una relación inversamente proporcional con
TCONTVO. Para la variable cualitativa se muestra el diagrama de medias en la Figura 6, donde se observa que tiene una relación con el NBI. GRPB fue el grupo que se tomó como referencia de comparación en el momento de realizar la variable dicótoma en el modelo. La mayoría de los municipios se encuentran con una generación insuficiente de recursos propios.

En el análisis espacial univariado se encuentra que todas las variables cuantitativas tienen autocorrelación positiva, según el índice de Moran y Geary; la Figura 7 muestra los dispersogramas correspondientes a cada variable, por lo cual fue necesario incluir el rezago espacial de cada una de ellas en el modelo.

Aunque la información de las variables utilizadas puede ser adquirida 


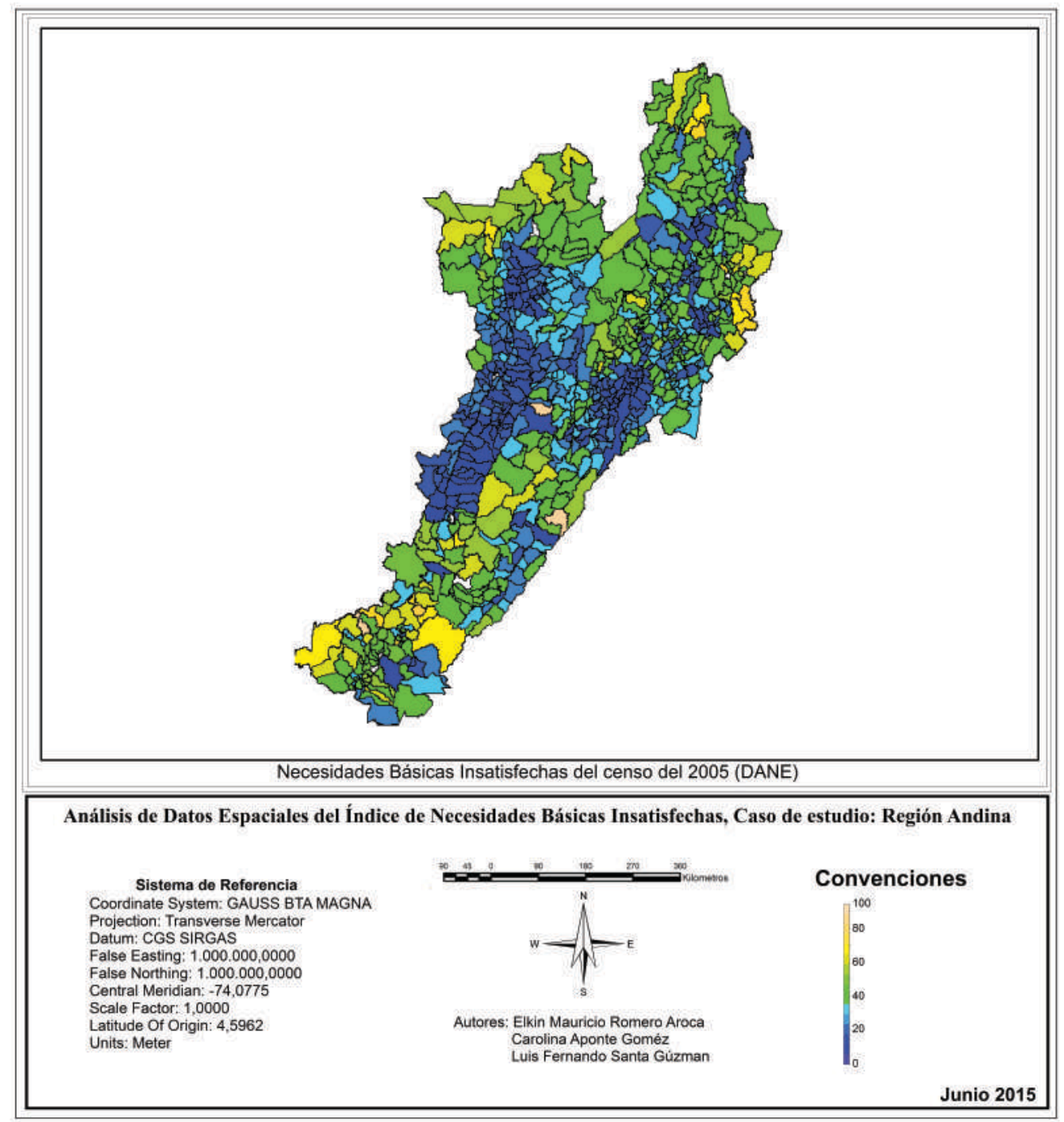

Figura 4. Mapa del Indice de Necesidades Basicas Insatisfechas Fuente: Elaboración propia

en diferentes entidades gubernamentales, es importante destacar que, al ser datos tomados externamente, la integridad de la información puede estar comprometida, dado que no se conoce su tratamiento, y, por ende, los resultados del modelo dependen de la forma como fue construida la base de datos.

\subsection{Estimación del Modelo}

El modelo inicial se escogió de acuerdo con el análisis de las fun- 

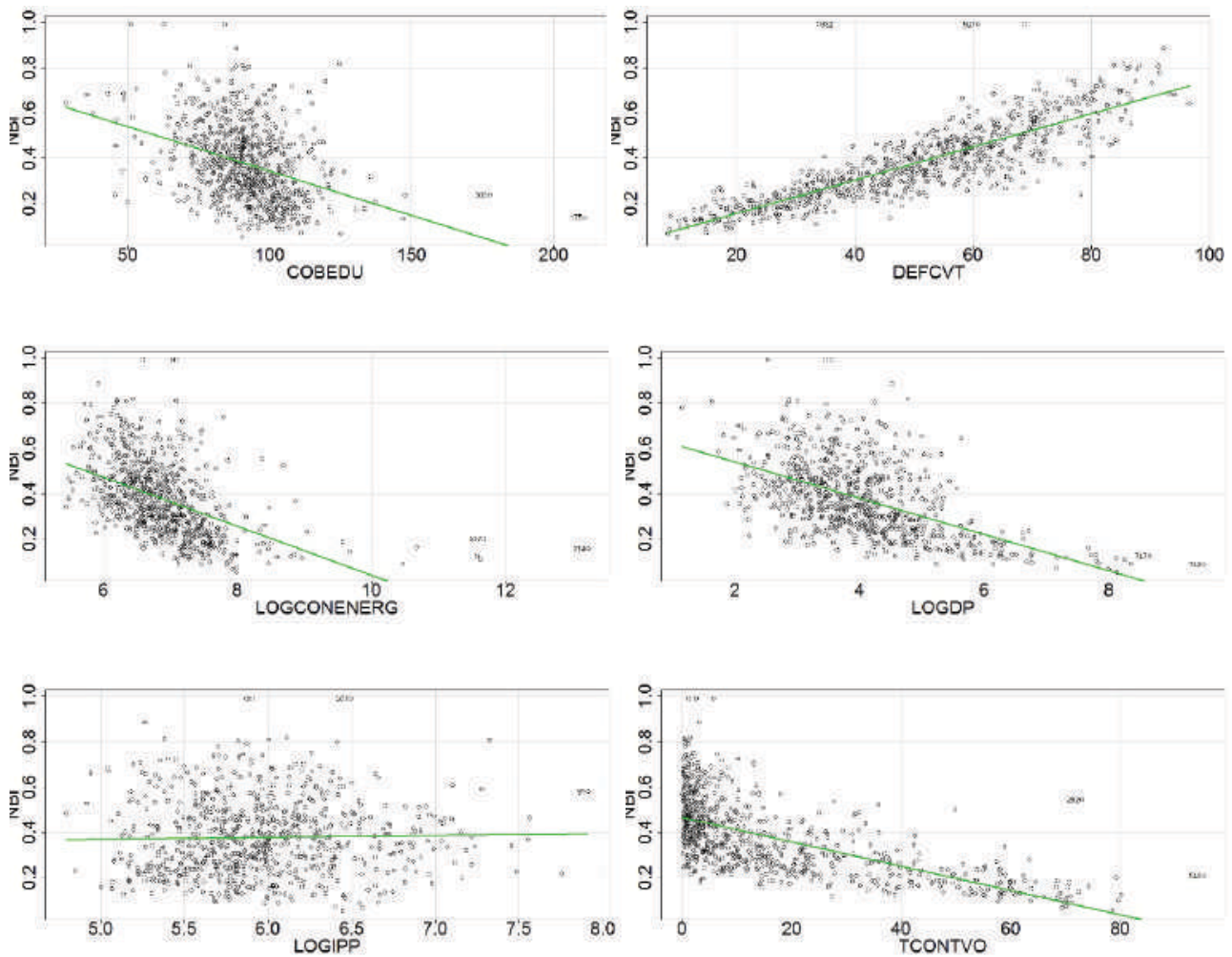

Figura 5. Diagramas de dispersión entre la variable dependiente e independientes Fuente: Elaboración propia

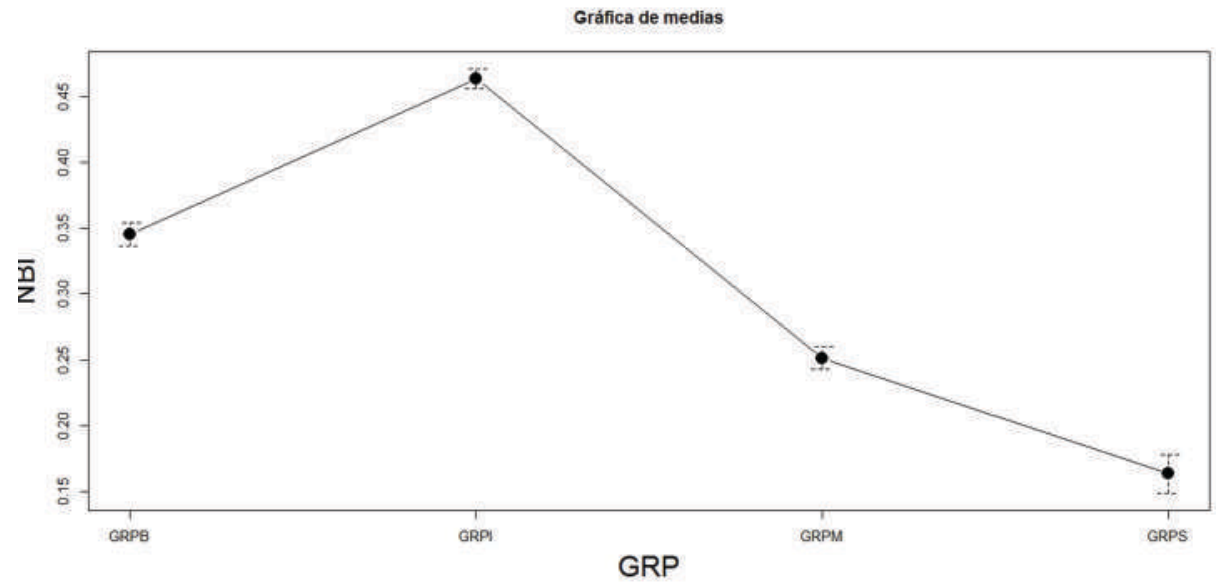

Figura 6. Diagrama de medias

Fuente: Elaboración propia 

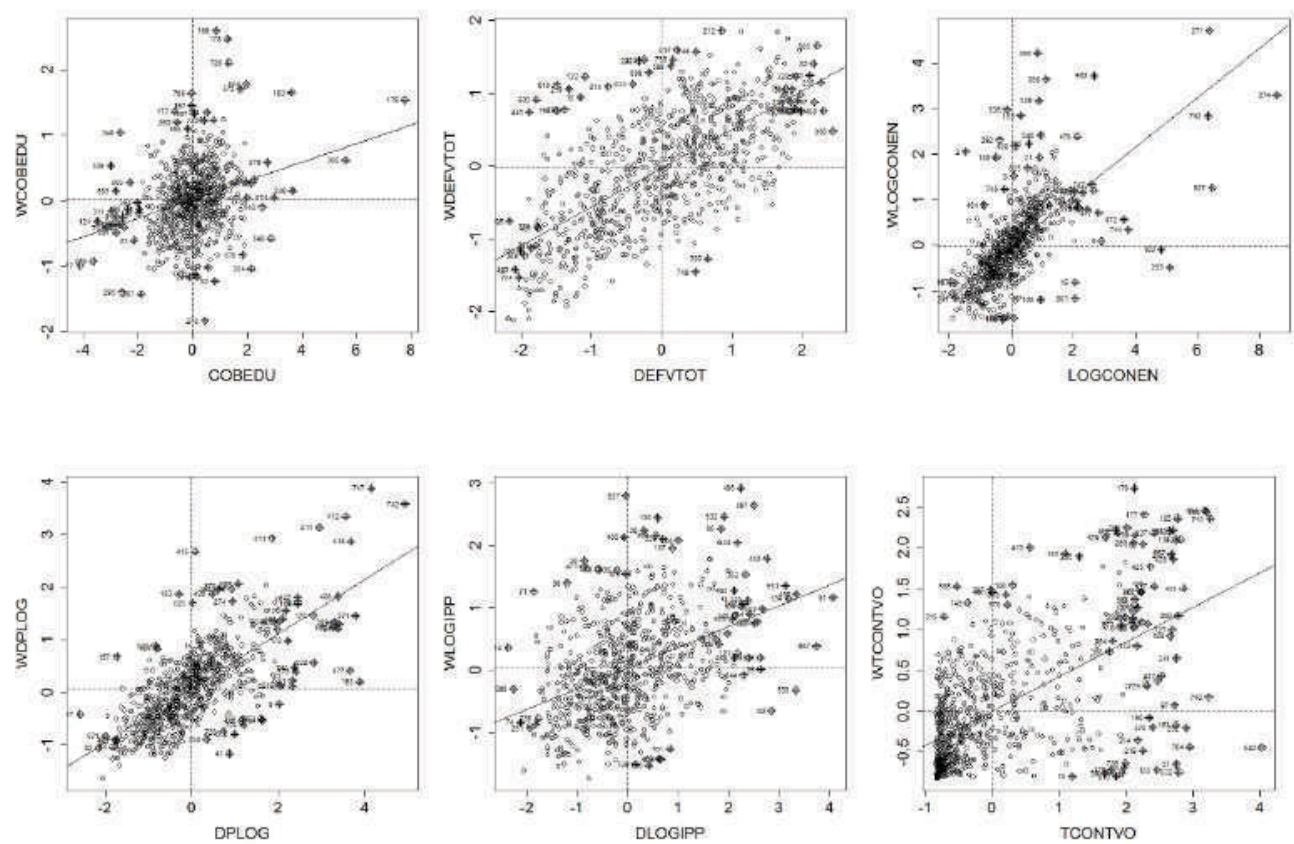

Figura 7. Dispersogramas de Moran

Fuente: Elaboración propia

ciones de enlace que son utilizadas en la generación de un modelo con regresión beta econométrico y espacial (Cribari-Neto \& Zeileis, 2010).
Estos modelos se diagnosticaron de acuerdo con el parámetro de precisión, el criterio BIC y el Pseudo R2 mostrados en la Tabla 3.

Tabla 3. Diagnósticos de selección para la función de enlace

\begin{tabular}{|l|r|r|r|}
\hline Función de enlace & \multicolumn{1}{|c|}{ Pseudo-R2 } & BIC & Parámetro de precisión \\
\hline Logit & 0,697 & $-1469,17$ & 26,27 \\
\hline Probit & 0,719 & $-1465,20$ & 26,14 \\
\hline log-log & 0,587 & $-1438,54$ & 25,24 \\
\hline clog-log & 0,783 & $-1480,82$ & 26,65 \\
\hline Cauchit & 0,138 & $-1472,50$ & 26,28 \\
\hline
\end{tabular}

Fuente: Elaboración propia

De esta manera, se encontró el modelo inicial a partir del criterio de Complemento Log-Log de la distri- bución Beta, dado por la función de enlace mostrada en (1): 
(1)

Se realizaron pruebas estadísticas basadas en el contraste de razón de verosimilitudes y en el criterio BIC, las cuales indicaron inicialmente que todas las variables, excepto LOGCPENR, fueron significativas. Este modelo sin efectos espaciales se muestra en la Tabla 4, indicando que el $78,3 \%$ de la variabilidad de la variable endógena es explicada por las variables exógenas (Melo, Oller, \& Cuyas, 2010). En segunda instancia, se evaluaron los supuestos del mo- delo, y se encontró que el factor de inflación de la varianza de las variables independientes era muy pequeño, $\mathrm{y}$, por lo tanto, no se encontraron problemas de multicolinealidad. El segundo supuesto de autocorrelación fue evaluado con el análisis espacial de los residuales ponderados estandarizados mostrados en la Figura 8, con los índices de autocorrelación espacial global y local de acuerdo con las pruebas de Moran y Geary, encontrando autocorrelación espacial en estos, como se muestra en la Tabla 4 , por lo que fue necesario hallar los retardos espaciales de cada variable exógena y la variable dependiente.
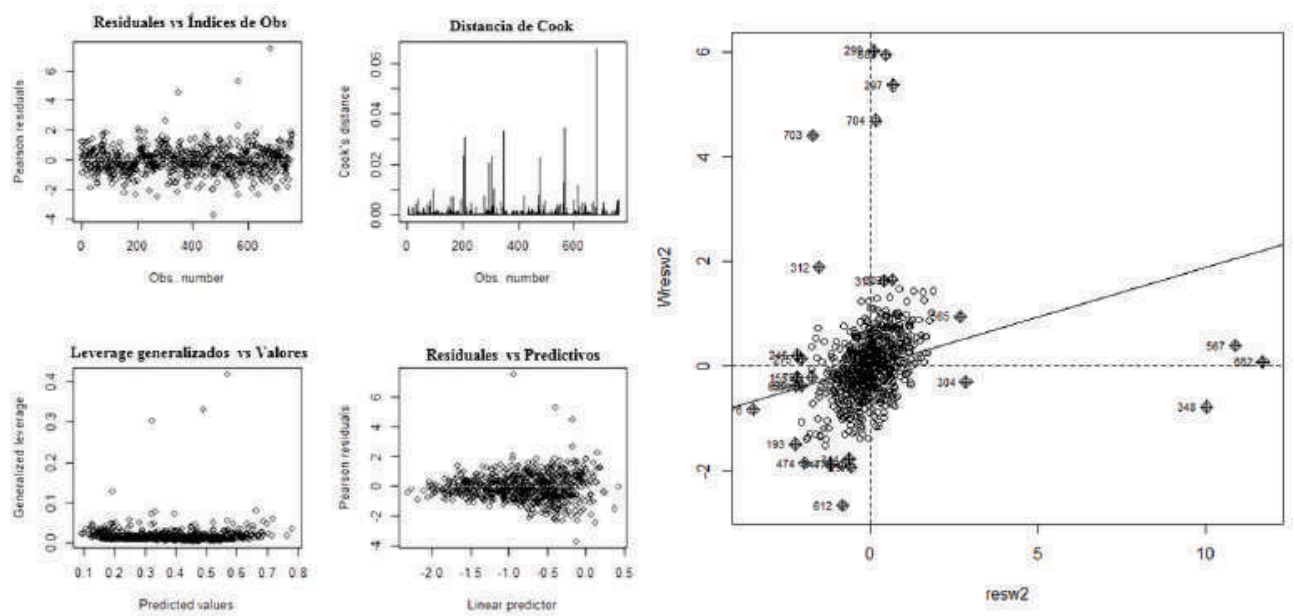

Figura 8. Análisis de residuales del modelo sin efectos espaciales Fuente: Elaboración propia 
Tabla 4. Diagnóstico de los modelos

\begin{tabular}{|c|c|c|c|c|c|c|c|}
\hline $\begin{array}{c}\text { Variables } \\
\text { independientes }\end{array}$ & Estimación & VIF & $\begin{array}{l}\text { Pseudo R- } \\
\text { cuadrado }\end{array}$ & $\mathrm{BIC}$ & $\begin{array}{l}\text { Parámetro de } \\
\text { precisión }\end{array}$ & $\begin{array}{c}\text { Índice de } \\
\text { Moran (SW2) }\end{array}$ & $\begin{array}{c}\text { Índice de } \\
\text { Geary (SW2) }\end{array}$ \\
\hline $\begin{array}{l}\text { (Intercept) } \\
\text { Econométrico }\end{array}$ & $-2,460^{* *}$ & & \multirow{11}{*}{0,783} & \multirow{11}{*}{$-1480,82$} & \multirow{11}{*}{$\begin{array}{c}26,65 \mathrm{p}- \\
\text { value }=2 \mathrm{e}-16\end{array}$} & \multirow{11}{*}{$\begin{array}{c}0,189 \mathrm{p}- \\
\text { value }=2 \mathrm{e}-16\end{array}$} & \multirow{11}{*}{$\begin{array}{c}0,769 \\
\text { p-value }= \\
0,003\end{array}$} \\
\hline COBEDU & $-0,003^{* * *}$ & 1,141 & & & & & \\
\hline DEFCVT & $0,020^{* * *}$ & 2,234 & & & & & \\
\hline GRPI & 0,059 & 2,927 & & & & & \\
\hline GRPM & 0,076 & & & & & & \\
\hline GRPS & $-0,007$ & & & & & & \\
\hline LOGIPP & $-0,080^{* *}$ & 1,122 & & & & & \\
\hline LOGCPENR & 0,010 & 1,660 & & & & & \\
\hline LOGDP & $-0,070^{* * *}$ & 1,607 & & & & & \\
\hline LOGMIM & $0,373^{*}$ & 1,573 & & & & & \\
\hline TCONTVO & $-0,003^{* *}$ & 2,802 & & & & & \\
\hline $\begin{array}{l}\text { (Intercept) } \\
\text { Espacial }\end{array}$ & $-1,443^{* * *}$ & & \multirow{17}{*}{0,8283} & \multirow{17}{*}{$-1593,32$} & \multirow{17}{*}{$\begin{array}{c}32,55 \mathrm{p}- \\
\text { value }=2 \mathrm{e}-16\end{array}$} & \multirow{17}{*}{$\begin{array}{c}0,019 p- \\
\text { value }=0,515\end{array}$} & \multirow{17}{*}{$\begin{array}{c}0,943 \\
\text { p-value }= \\
0,518\end{array}$} \\
\hline WNBI & $1,749 * * *$ & 3,143 & & & & & \\
\hline COBEDU & $-0,002^{* * *}$ & 1,193 & & & & & \\
\hline WCOBEDU & $-0,002^{* *}$ & 1,419 & & & & & \\
\hline DEFCVT & $0,016^{* * *}$ & 2,853 & & & & & \\
\hline WDEFCVT & $-0,002$ & 3,774 & & & & & \\
\hline LOGDP & $-0,051^{* *}$ & 2,894 & & & & & \\
\hline WLOGDP & $-0,025$ & 2,761 & & & & & \\
\hline TCONT & $-0,004^{* *}$ & 3,082 & & & & & \\
\hline WTCONTVO & $0,003^{*}$ & 3,001 & & & & & \\
\hline GRPI & $0,070^{*}$ & 2,730 & & & & & \\
\hline GRPM & 0,044 & & & & & & \\
\hline GRPS & $-0,031$ & & & & & & \\
\hline LOGIPP & $-0,041$ & 1,343 & & & & & \\
\hline WLOGIPP & $-0,012$ & 1,329 & & & & & \\
\hline LOGCPEN & $-0,014$ & 2,448 & & & & & \\
\hline WLOGCPENR & 0,051 . & 2,366 & & & & & \\
\hline \multicolumn{8}{|c|}{ *** Parámetro significativo entre 0\% y 0,1\%. } \\
\hline \multicolumn{8}{|c|}{ *** Parámetro significativo entre 0,1\% y 1\%. } \\
\hline \multicolumn{8}{|c|}{ * Parámetro significativo entre 1\% y 5\%. } \\
\hline Parámetro signific & entre $5 \%$ y $1 c$ & & & & & & \\
\hline
\end{tabular}


Para realizar el modelo espacial de cada una de las variables y, de regresivo autorregresivo espacial, esta manera, se estimó el nuevo se plasmó la ubicación de las coor- Modelo de Regresivo Autorregresivo denadas representativas de los 760 Espacial de Tipo Beta, en el cual fue municipios, como se muestra en el necesario retirar la variable MIM Figura 9; a partir de esas coordena- por no resultar significativa. das fueron encontrados los retardos

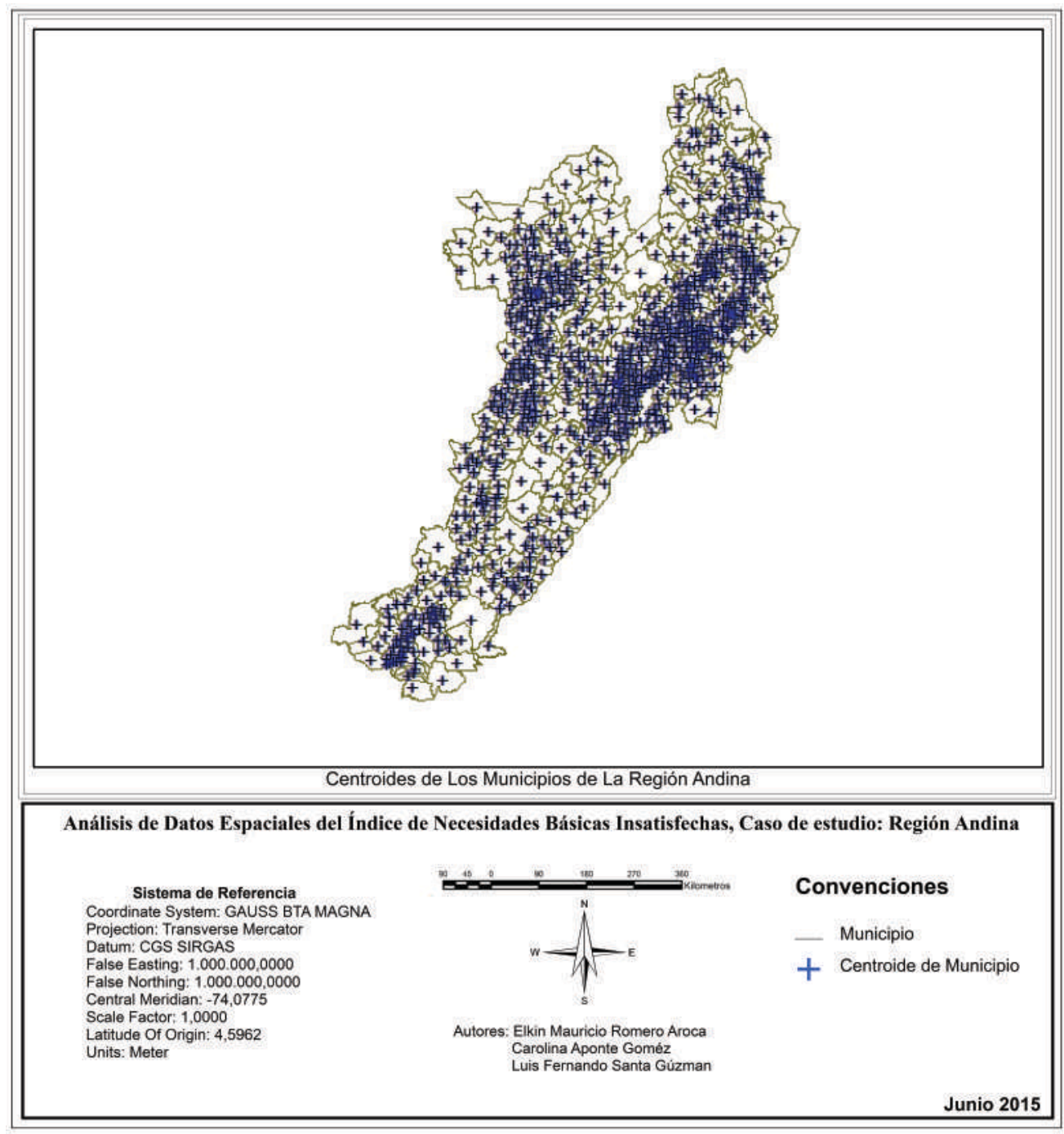

Figura 9. Centroides de los municipios de la región Andina

Fuente: Elaboración propia 
De acuerdo con los parámetros establecidos por Stiglitz, Sen y Fitoussi en el informe de la Commission on the Measurement of Economic Performance and Social Progress, deben existir ocho dimensiones mínimas que se han de considerar simultáneamente para la medición del bienestar de una población (Angulo, Diaz, \& Pardo, 2012). En el modelo final realizado se encuentran presentes seis de estas dimensiones, representadas de la siguiente manera: LOGCPEN en material para una vida estándar (ingreso, consumo y riqueza), TCONTVO en salud, COBEDU en educación, IPP en actividades personales (incluyendo el trabajo), GRP en voz política y gobernabilidad y, por último, DEFCVT en seguridad económica y física. Bajo estos parámetros no fue posible encontrar variables que representaran las condiciones ambientales y conexiones sociales de cada municipio.

Aunque la densidad poblacional no se encuentre enmarcada sobre las dimensiones mínimas anteriormente mencionadas, es importante destacar que esta es sustancial para la determinación de la pobreza, ya que si un territorio cuenta con altos recursos y producción, puede sostener una población muy grande, pero si tiene alta densidad y bajos ingresos, puede generar mayor pobreza en la región (Alonso, 2006). Por esta razón fue tomada en cuenta como variable independiente $\mathrm{y}$, además, resultó significativa en el modelo.
Desde hace 10 años no se genera una actualización de la información con la que se obtiene el índice de NBI (DANE, 2006), por lo que contar con factores que determinen el nivel de pobreza, que puedan ser medidos de manera más sencilla y reflejen las transformaciones que vive el país, reducirá el tiempo de evaluación de cada municipio y, por ende, de la destinación de los recursos; esto lleva a que los planes de desarrollo ejecutados sean eficaces y eficientes en la disminución de la situación de pobreza, realzando la importancia de los programas sociales del gobierno (Baltazar, Grillo, \& Karpf, 2007).

El Modelo Mixto Regresivo Autorregresivo Espacial de Regresión Beta final se encuentra en la Tabla 4, donde se incluyen los rezagos espaciales de las variables exógenas, el rezago de la variable endógena y las variables exógenas iniciales; este indica que ahora el 82,83\% del comportamiento del índice NBI es explicado por las variables exógenas. Al realizar el análisis de los residuales ponderados estandarizados de dicho modelo se pudo comprobar que la dependencia espacial fue eliminada, indicando que no hay evidencia estadística suficiente para rechazar la hipótesis nula de las pruebas del índice de Moran y de Geary ( ; ); esto se construyó utilizando la selección de la matriz de vecindad mediante el método de coordenadas principales 
de las matrices de pesos espaciales (PCNM), dando como resultado el criterio de contigüidad de dos vecinos más cercanos, el cual sirvió para realizar la descomposición espectral de la matriz de pesos espaciales y garantizar que los vectores propios maxi- mizaran el criterio de autocorrelación global de Moran I(x). En la Figura 10 se muestra la corrección del supuesto; dado que su pendiente es muy baja, se infiere la baja autocorrelación entre los datos.

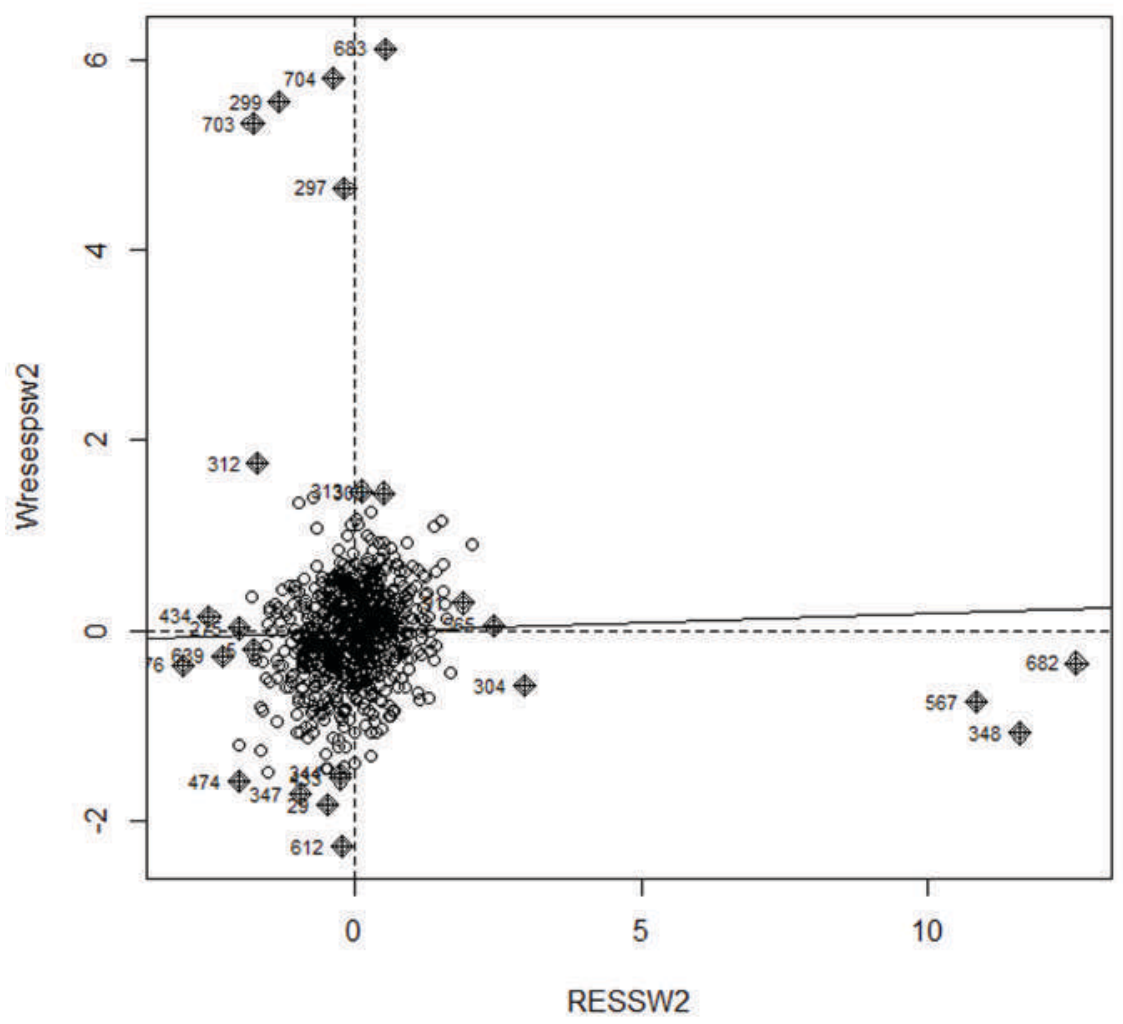

Figura 10. Dispersograma de Moran de los residuales del modelo espacial Fuente: Elaboración propia

Adicionalmente, se realizaron de nuevo los indicadores locales de autocorrelación espacial, como se muestra en la Figura 11, donde se evidencia la falta de aglomeraciones en su distribución y eliminando la presencia del efecto de dependencia espacial en el modelo. Algunos municipios aún sobresalen, siendo estos indicadores de valores significativos en la variable respuesta, haciendo que los municipios vecinos presenten valores simila- 
res a ellos; entre los puntos calientes se encuentran Murillo-Tolima, Villa Hermosa-Tolima, Gachalá-Cundinamarca, Gama-Cundinamarca, Vetas-Santander y Mutiscua-Norte de Santander, con valores alto-alto, y Miranda-Cauca, Tello-Huila, ChitaBoyacá y Beteitiva-Boyacá, con valores bajo-bajo.

Se evidencia la autocorrelación en los municipios de acuerdo con la significancia del rezago de la variable respuesta, con un valor positivo de 1,749 , que implica la existencia de un efecto de agregación espacial, demostrando la relación en el comportamiento geográfico de estos y cómo este afecta la pobreza de cada uno, pues los municipios con mayor índice de NBI se encuentran rodeados de municipios con alta pobreza, y municipios con baja pobreza se rodean de municipios con bajos niveles de $\mathrm{NBI}$; comprobando la primera ley de la geografía, que indica que "Todas las cosas están relacionadas entre sí, pero las cosas más próximas en el espacio tienen una relación mayor que las distantes".

Se efectuó el análisis de los residuales, como se muestra en la Figura 11, para encontrar las observaciones atípicas e influyentes. Se encontraron 35 valores Hat y 15 puntos de Leverage generalizados, de los cuales sobresalen los municipios de Baraya-Huila y Cumbitara-Nariño, con un valor del índice NBI de 100\%; municipios que se pueden considerar centros de periferia, ya que a su alrededor se encuentran municipios con alto índice de NBI; aunque Anzoátegui-Tolima cuenta con el mismo valor de NBI, se encuentra rodeado de municipios con un bajo nivel de pobreza. El comportamiento de estos tres municipios es atípico, frente al general de la región, por lo indicado anteriormente en el análisis univariado. Además, se encuentra también el municipio de Jamundí-Valle del Cauca, que tiene un bajo índice de NBI, pero el consumo de energía de sus habitantes lo hace atípico, debido a que es el más alto de toda la región, con un valor de $554.666 \mathrm{~kW} / \mathrm{hab}$., siendo 1.710,29 $\mathrm{kW} / \mathrm{hab}$ el promedio total.

Por último, se compararon los valores observados de la variable endógena y los estimados en el modelo, para verificar su concordancia, y se evidenció que el modelo refleja la situación del índice de NBI, por la alta coincidencia entre los dos valores mostrados en la Figura 12. Los valores intermedios se encuentran concentrados en la zona norte de la región, mientras que los municipios con bajas NBI se encuentran en la parte central y occidental, coincidiendo con la ubicación de los departamentos de Cundinamarca, Valle del Cauca y Antioquia, donde se encuentran las tres capitales más importantes de la región. Además, 


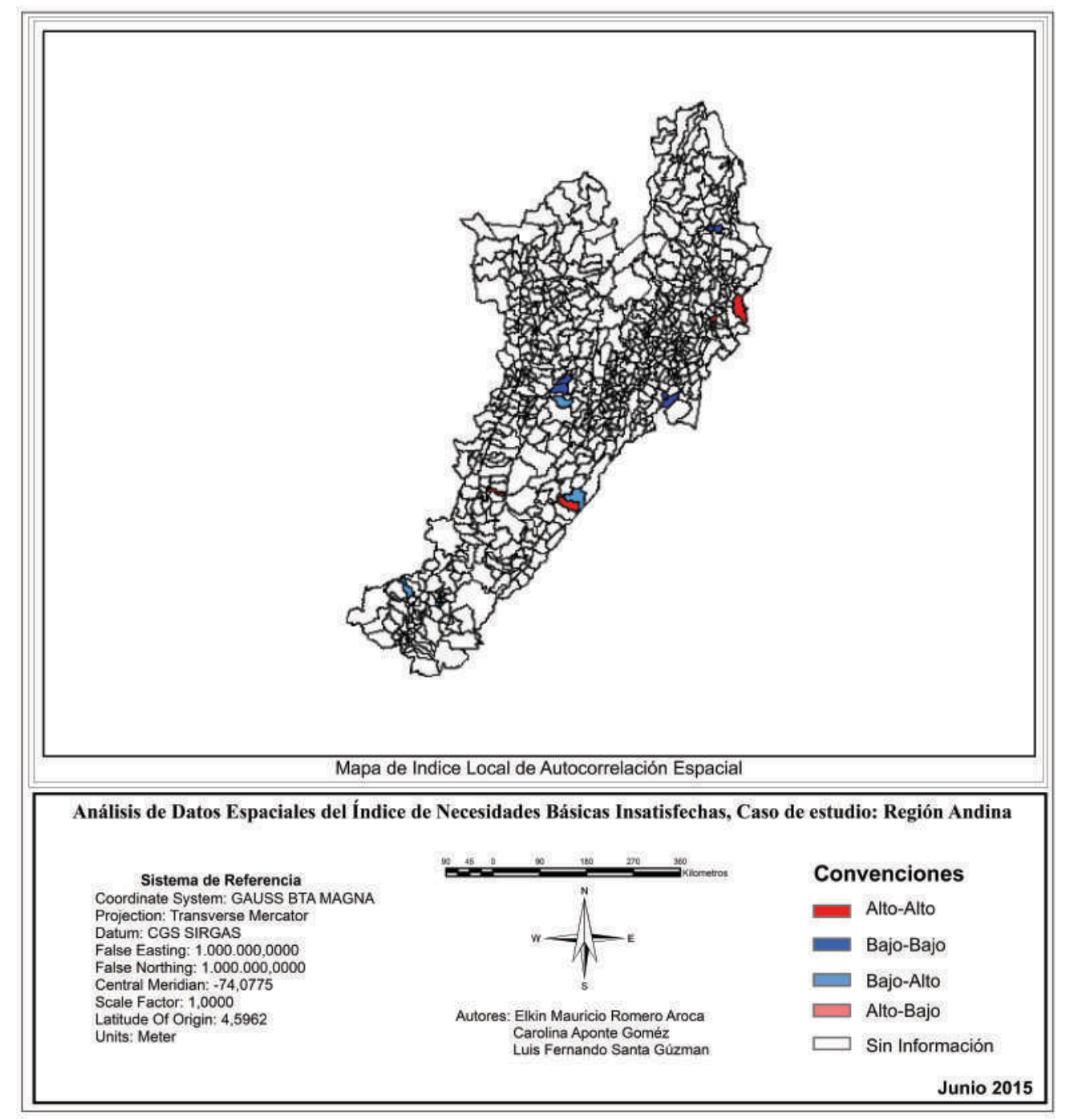

Figura 11. Mapa LISA de los residuales ponderados estandarizados del modelo espacial Fuente: Elaboración propia

la mayoría de los municipios que cuentan con altos niveles de NBI se concentran al sur y nororiente, en los departamentos de Nariño, Boyacá y Cauca, coincidiendo con el análisis realizado al mapa LISA de los residuales del modelo.
Popayán y Pasto representan una situación atípica en la distribución del índice NBI, como se muestra en la Figura 13, ya que cuentan con niveles bajos de pobreza, pero los municipios a su alrededor presentan una situación contraria. Analizando los valores de 

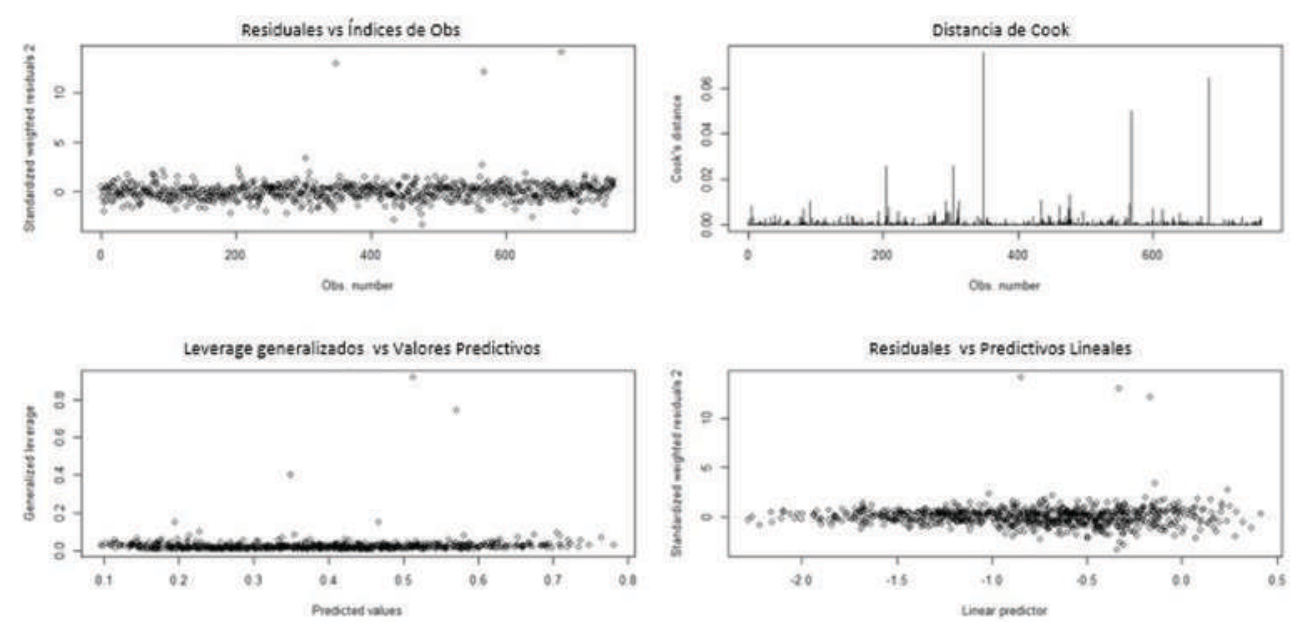

Figura 12. Análisis residuales modelo espacial Fuente: Elaboración propia

las variables independientes del modelo en Popayán y Pasto, se encontró que los dos municipios presentan un alto índice de afiliación al régimen contributivo, en comparación con los municipios aledaños, de lo que se puede inferir que la cantidad de personas laboralmente activas influye en el nivel de pobreza de una población. También se muestra que otra variable significativa para estos dos municipios es la cobertura de educación, pues presentan un nivel medio-alto de acceso, superior al promedio.

Se debe entender la importancia que para un país tiene determinar las NBI de la población, dado que indican problemáticas que deben ser solucionadas mediante decisiones y medidas gubernamentales pertinentes que ofrezcan a los habitantes de una región mejores condiciones de vida.
Con la Constitución de Colombia de 1886 se presentaban complicaciones en la distribución de los recursos para el financiamiento de las entidades territoriales, ya que el $30 \%$ de los dineros corrientes de la nación eran distribuidos en igual proporción entre todas las entidades territoriales del país, y el restante $70 \%$, en proporción con su población; dicha metodología generó focos de intereses políticos y un detrimento en los municipios con mayor índice de NBI. La situación cambio con la Constitución de 1991, al hacer uso del índice de NBI y de algunas otras variables, renovando los métodos utilizados y haciéndolos más precisos, logrando obtener mejores políticas para el bienestar de la población (Pérez, 1994).

Actualmente, el índice de NBI es utilizado en diferentes procesos, como el 




Figura 13. Comparación entre los valores del índice NBI y los predictivos del modelo Fuente: Elaboración propia

Sistema Nacional de Participaciones, regulado por la Ley 715 de 2001, y el Sistema General de Regalías, reglamentado por la Ley 1530 de 2012. Esto significa que los recursos son repartidos según el nivel de pobreza relativa, que se destinan más ingresos para los departamentos que presentan mayor índice de NBI, mejorando los objetivos de este sistema para impulsar el crecimiento, la equidad, la reducción de índices de pobreza y competitividad de las regiones (Bonet \& Urrego, 2014). 
Uno de los indicadores más importantes para la elaboración y desarrollo de proyectos y programas en los planes de desarrollo nacionales, departamentales y municipales es el de NBI; esto en razón del artículo 366 de la Constitución Política de Colombia, que promulga:

El bienestar general y el mejoramiento de la calidad de vida de la población son finalidades sociales del Estado. Será objetivo fundamental de su actividad la solución de las necesidades insatisfechas de salud, de educación, de saneamiento ambiental y de agua potable. Para tales efectos, en los planes y presupuestos de la Nación y de las entidades territoriales, el gasto público social tendrá prioridad sobre cualquier otra asignación.

Además, el artículo 324 indica:

Las juntas administradoras locales distribuirán y apropiarán las partidas globales que en el presupuesto anual del Distrito se asignen a las localidades teniendo en cuenta las necesidades básicas insatisfechas de su población.

Lo cual es reglamentado por la Ley 152 de 1994, que en el literal e) del artículo tercero indica:

Prioridad del gasto público social. Para asegurar la consoli- dación progresiva del bienestar general y el mejoramiento de la calidad de vida de la población, en la elaboración, aprobación y ejecución de los planes de desarrollo de la Nación y de las entidades territoriales se deberá tener como criterio especial en la distribución territorial del gasto público el número de personas con necesidades básicas insatisfechas, la población y la eficiencia fiscal y administrativa, y que el gasto público social tenga prioridad sobre cualquier otra asignación.

De acuerdo con el índice de NBI se distribuye el gasto público social y se toman decisiones para enfocar los recursos a un tema específico, pero, como se ha indicado anteriormente, este índice es actualizado en periodos muy largos, por lo cual tomar decisiones con base en él no es eficiente ante la situación actual de la población, y la desigualdad de las regiones se hace persistente.

\section{Conclusiones}

Se logró desarrollar un modelo mixto de regresión beta regresivo autorregresivo espacial que determina el índice de NBI en la Región Andina, según el censo de 2005, estimando los parámetros del modelo por medio de técnicas estadísticas y de análisis espacial; además, el modelo presenta 
una bondad de ajuste, y el comportamiento del índice de NBI es explicado por las variables independientes.

Se encontró que las variables que posiblemente pueden explicar el índice de NBI sin la necesidad de elaborar un censo a nivel nacional son cobertura en educación, déficit de vivienda total, densidad poblacional, tasa de afiliación al régimen contributivo, ingreso público per cápita, generación de recursos propios y consumo promedio de energía por habitante. Es importante destacar que fue ne- cesario incluir el retardo espacial en todas las variables del modelo, ya que al realizar las pruebas de autocorrelación, todas presentaban dependencia espacial; la inclusión de esta solución fortaleció la estimación del modelo y los análisis realizados al índice de Necesidades Básicas Insatisfechas de la Región Andina.

\section{Agradecimientos}
A la Universidad Distrital Francisco José de Caldas, por su respaldo en la elaboración de este artículo.

\section{Referencias}

Alkire, S., \& Foster, J. (2011). Understandings and Misunderstandings of Multidimensional. $\mathrm{N}^{\circ}$ 43. Journal of Public Economics, 476-489. .

Alonso, I. (2006). Demografía y pobreza, de Malthus a nuestros días. Perspectiva, 54-57.

Andrade, G., Gonzalez, V., Cardenas, D., Gómez, S., Ocampo, J., Lozano, B., . . . Santos, E. (2003). Enciclopedia Colombia a su alcance Tomo I. Bogotá D.C.: Planeta Colombiano S.A.

Angulo, R. C., Diaz, B. Y., \& Pardo, R. (2012). Multidimensional Poverty in Colombia, 1997-2010. Institute of Social and Economic Research, 1-54.

Baltazar, E. N., Grillo, S., \& Karpf, E. (2007). ¿Cuál es el mejor indicador de pobreza en Colombia para la orientación del gasto público social?. Vol. 12, $\mathrm{N}^{\circ} 1$. Revista SciELO, 117-144.

Benson, T., Chamberlin, J., \& Rhinehart, I. (2005). An investigation of the spatial determinants of the local prevalence of poverty in rural Malawi. Vol. 30. Food Policy, 532-550.

Bonet, J., \& Urrego, J. (2014). El Sistema General de Regalías: ¿mejoró, empeoró o quedó igual? Cartagena: Banco de la República.

Bourguignon, F., \& Chakravarty, S. (2003). The measurement of multidimensional poverty. Vol 1. Journal of Economic Inequality, 25-49.

Canavos, G. (1988). Probabilidad y estadística: Aplicaciones y Métodos. México: McGraw-Hill.

Constitución Politica de Colombia. (1991). Asamblea Nacional Constituyente. Bogotá D.C., Colombia. 
Cribari-Neto, F., \& Zeileis, A. (2010). Beta Regression in R. Vol. 34. Journal of Statistical Software, 1-21.

DANE. (2006). Boletín - Censo General de Necesidades Básicas Insatisfechas. Obtenido de Departamento Administrativo Nacional de Estadística: https:/www.dane.gov.co/censo/ files/resultados/prest_NBI_100708.pdf

Dobson, A. J. (2002). An introduction to generalized linear models. Florida: Chapman \& Hall/CRC.

Feres, J., \& Mancero, X. (2001). El método de las necesidades básicas insatisfechas (NBI) y sus aplicaciones en américa latina. Naciones Unidas-CEPAL-División estadística y Proyecciones Económicas, 61-100.

Fernandez, E. A. (2008). Análisis de Regresión. Mayagüez: Edgar Acuña.

Ferrari, S., \& Cribari-Neto, F. (2004). Beta Regression for Modelling Rates and Proportions. Journal of Applied Statistic, 799-815.

Fresneda, O. (2007). La medida de necesidades básicas insatisfechas (NBI) como instrumento de medición de la pobreza y focalización de programas. $\mathrm{N}^{\circ} 18$. Estudios y perspectivas , 1-52.

González, J. I. (2011). Utilitarismo y Mediciones de Pobreza. Vol. 13, № 25. Economía Institucional, 89-103.

González, J., \& Daffara, C. (Abril de 2000). Free Software / Open Source: Information Society Opportunities for Europe? Obtenido de European working group on libre software : http://eu.conecta.it/

Gujarati, D. N., \& Porter, D. C. (2010). Econometría. México: McGraw-Hill Irwin.

IGAC. (2008). Sistema de Información Geográfica para la Planeación y el Ordenamiento Territorial. Obtenido de http://sigotn.igac.gov.co/sigotn/

Lance, W., \& Carol, G. (2004). Applied Spatial Statistics for Public Health Data. New York: Wiley.

Ley $\mathrm{N}^{\circ}$ 152. (15 de Junio de 1994). Congreso de Colombia. Diario Oficial 41450. Bogotá D.C., Colombia.

Ley $\mathrm{N}^{\circ}$ 1530. (17 de Mayo de 2012). Congreso de Colombia. Diario Oficial 48433. Bogotá D.C., Colombia.

Ley $N^{\circ}$ 715. (21 de Diciembre de 2001). Congreso de Colombia. Bogotá D.C., Colombia.

Melo, O., Oller, J., \& Cuyas, F. (2010). Análisis de regresión beta a través de distancias. Cataluña: Universitat Politécnica de Catalunya.

Montgomery, D. C., \& Runger, G. C. (2002). Probabilidad y estadística aplicadas a la ingeniería. México: Editorial Limusa.

Noel, C. (1993). Statistics for Spatial Data. New York: Wiley-Interscience.

O’Sullivan, D., \& Unwin, D. (1943). Geographic Information Analysis. The Pennsylvania State University, 315-355.

Peña, D. (2002). Análisis de Datos Multivariantes. Madrid: MCGRAW-HILL S.A.

\begin{tabular}{l|l}
\hline 418 & Carolina Aponte Gómez, Elkin Mauricio Romero Aroca, Luis Fernando Santa Guzmán
\end{tabular} 
Pérez, I. J. (1994). Diez años de descentralización : resultados y perspectivas. Bogotá D.C.: Kinte.

Rupasingha, A., \& Goetz, S. J. (2007). Social and political forces as determinants of poverty:A spatial analysis. Vol. 36. The Journal of Socio-Economics, 650-671.

Salazar, I. (2010). Geografía económica de la Región Andina Oriental. Cartagena de Indias,: Banco de la República.

Schabenberger, O., \& Gotway, C. (2004). Statistical methods for spatial data analysis. CRC press, 23-86.

Sen, A. (1976). Poverty: An Ordinal Approach to Measurement. Vol. 44, № 2. Econometrica, 219-231.

Valcarce, E. V., \& Serrano, R. M. (2000). Técnicas econométricas para el tratamiento de datos espaciales: La econometría espacial. $N^{\circ} 1$. Barcelona: Universidad de Barcelona.

Wooldridge, J. (2010). Introducción a la econometría Un enfoque moderno. México Distrito Federal: Cengage Learning. 
Recepción:

Evaluación:

Aprobación:

\begin{tabular}{l|l}
\hline 420 & Carolina Aponte Gómez, Elkin Mauricio Romero Aroca, Luis Fernando Santa Guzmán
\end{tabular} 